\title{
Radiologic analysis of correlations between sinonasal anatomical variations in patients with nasal septal deviation
}

\author{
Elif Karalı ${ }^{1}$, Akif Güneş ${ }^{1}$, Emine Dağıstan ${ }^{2}$, Zeliha Coşgun ${ }^{2}$, Ahmet Ural $^{1}$, Oya Kalaycıoğlu ${ }^{3}$ \\ ${ }^{1}$ Department of Otorhinolaryngology, Bolu Abant Izzet Baysal University Medical Faculty, Bolu, Turkey \\ ${ }^{2}$ Department of Radiology, Bolu Abant Izzet Baysal University Medical Faculty, Bolu, Turkey \\ ${ }^{3}$ Department of Bioistatistics and Medical Information, Bolu Abant Izzet Baysal University Medical Faculty, Bolu, Turkey
}

Received: 24 October 2020, Accepted: 04 December 2020, Published online: 31 December 2020

(C) Ordu University Institute of Health Sciences, Turkey, 2020

\begin{abstract}
Objective: This study aims to analyze the sinonasal anatomical variations accompanying the nasal septal deviation, the correlations between these variations, and their relationship with the septal deviation angle.

Methods: In this retrospective study, preoperative paranasal computed tomography (CT) scans of 206 patients who underwent septoplasty between January 2015 and December 2019 were examined. In CT scans, different nasal septal deviation types, Keros classification, optic nerve type, ethmoid air cell variants, nasal concha variants, paranasal sinus pneumatization variants, and the correlation between accessory pneumatization variants and their relationship with the septal deviation angle were analyzed.

Results: In patients with nasal septal deviation, supraorbital ethmoid cell, anterior clinoid process pneumatization and onodi cell were more frequent compared to the literature. Any significant correlation between the nasal septal deviation angle and the presence of sinonasal variants was not detected $(p>0.05)$. Correlations were significant between the presence of Frontal sinus hypoplasia and Haller cell $(\phi=-0.142, p$ $=0.042)$, Supraorbital Ethmoid cell $(\phi=-0.173, \mathrm{p}=0.013)$ and Paradoxal middle concha $(\phi=0.152, \mathrm{p}=$ 0.029 ).

Conclusion: Careful examination of paranasal CTs before craniomaxillofacial surgeries is important to determine sinonasal anatomic variants, to determine the appropriate treatment plan and to prevent possible complications peroperatively.

Key words: Nasal septal deviation, Paranasal computerized tomography, Anatomic variation, Supraorbital ethmoid cell, Anterior clinoid process

Suggested Citation: Karali E, Gunes A, Dagistan E, Cosgun Z, Ural A, Kalaycioglu O. Radiologic analysis of correlations between sinonasal anatomical variations in patients with nasal septal deviation. Middle Black Sea Journal of Health Science, 2020; 6(3):340351.
\end{abstract}

Address for correspondence/reprints:

E-mail: elifkarali8181@gmail.com

DOI: $\quad 10.19127 / \mathrm{mbsjohs.815984}$

Elif Karali

Telephone number: +90 (374) 25346563355

ORCID-ID 0000-0002-8164-4056 


\section{Introduction}

Nasal septal deviation (NSD) is one of the major causes of nasal obstruction. Paranasal sinus (PNS) computed tomography (CT) is a widespread method to assess the paranasal anatomy (Wuister et al., 2014). Nasal anatomy and accompanying sinonasal pathologies can be evaluated with preoperative PNS CT in patients undergoing septoplasty (Akoglu et al., 2007). There are many sinonasal variants, and the identification of sinonasal anatomic variants in paranasal CT scans before craniomaxillofacial surgeries are important to prevent complications. The most common sinonasal anatomical variations are Agger nasi cells, infraorbital ethmoidal cells (Haller), sphenoethmoidal cells (Onodi), NSD and concha bullosa (Kantarci et al., 2004; Alkire and Bhattacharyya, 2010). Sinonasal anatomical variants as upper concha bullosa, lower concha bullosa, uncinate bulla, supraorbital ethmoid cell, anterior clinoid process pneumatization (ACPP) are less rarely seen (Fadda et al., 2012).

According to Mladina's classification, NSD was categorized into seven types. Type 1, unilateral vertical crest up to nasal valve; Type 2, unilateral vertical crest reaching to nasal valve; Type 3, unilateral vertical crest at the level of middle nasal concha; Type 4, the combination of Type 1 defined as $\mathrm{S}$ type septal deviation or Type 2 combined with Type 3; Type 5, unilateral horizontal crest in touch with the lateral nasal wall; Type 6, bilateral horizontal crest, and Type 7, NSDs with combinations of all these types.

Delano classification was used to determine the relationship of the optic nerve to the sphenoid sinus (SS) (DeLano et al., 1996). According to this classification, there is no recess into the Type $1 \mathrm{SS}$ wall. Type 2 has a recess in the SS wall that does not come into contact with the posterior ethmoid cell. There is a protrusion to the SS wall in Type 3. Type 4 extends into the SS and posterior ethmoid cells.

The olfactory fossa depth was calculated by Keros classification. Accordingly, the length of the vertical line drawn from the horizontal line connecting the infraorbital nerves perpendicularly to the medial point of the ethmoid roof was determined as the height of the medial ethmoid roof. The length of the vertical line drawn from the horizontal line connecting the infraorbital nerves perpendicularly to the lamina cribrosa was determined as the height of the cribriform plate. The difference between the two heights was accepted as the height of the lamina lateralis, and the depths of olfactory fossa ranging between 1-3 mm, 4-7 mm, and 8-16 mm were graded as Keros Types 1, 2, and 3 .
The frequency of coexistence of NSD and concha bullous has been explored many times before (Koo et al., 2017; El-Taher et al., 2019). There are limited number of studies investigating the relationship between NSD and other sinonasal anatomical variations (Koo et al., 2017; Yazici, 2019).

This study aims to analyze the sinonasal anatomical variations accompanying the nasal septal deviation, the correlations between these variations, and their relationship with the septal deviation angle.

\section{Methods}

In our study, age, gender, and preoperative PNS CTs of 206 patients who underwent septoplasty between January 2015 and December 2019 were retrospectively analyzed. Patients younger than 18 years old, patients who had undergone septum or paranasal sinus surgery, patients with acute or chronic sinusitis, and patients with sinonasal polyposis or tumor were excluded from the study.

CT scans were obtained in the supine position using routine PNS CT imaging without contrast or sedation. CT examinations were performed by using a 64-channel multidetector CT unit (Revolution CT GE Healthcare, Milwaukee, WI, USA). The imaging parameters were KvP: 100, mAs: 90, a slice thickness $0.625 \mathrm{~mm}$, a reconstruction increment 1,5 $\mathrm{mm}$, a scan field of view of $20 \mathrm{~cm}$, and a matrix of $512 \times 512$ with the high-resolution bone algorithm.

CT scans were examined by a single experienced radiologist using PACS (Picture Archiving and Communicating System). Different NSD types, Keros classification, optic nerve type, ethmoid cell variants as Agger nasi cell, Onodi cell, Haller cell, supraorbital ethmoid cell, also nasal concha variants as superior concha bullosa middle concha bullosa, paradoxal middle concha, paranasal sinus pneumatization variants as frontal sinus hypoplasia and maxillary sinus hypoplasia; accessory pneumatization variants, as uncinate bulla in paranasal CTs; prevalence of ACPP and their inbetween correlations with NSD angle were evaluated.

In CT scans, two different classifications defined by Elahi et al. and Mladina were used to determine the type of septum deviation of patients (Elahi et al., 1997; Mladina et al., 2008). NSD was classified in 3 types as mild $\left(<9^{\circ}\right)$, medium $\left(9^{\circ}-15^{\circ}\right)$, advanced $(>$ $15^{\circ}$ ) according to the septal deviation angle (SDA rating system of Elahi et al. (Elahi et al., 1997) 


\section{Statistical Analyses}

The variables were summarized with median (Interquartile range) and frequency (percentage). Comparative statistical tests between groups were performed using the Kruskal-Wallis, Mann Whitney U, Pearson's chi-square or Fisher's exact tests, where appropriate. The $\phi$ (phi) correlation coefficient was used to estimate the degree of association between the anatomical variations. Statistical significance was set at $\mathrm{p}<0.05$ and the Statistical Package for Social Sciences 25.0 for Windows (SPSS Inc., Chicago, Illinois, USA) was used for conducting the analysis.

\section{Results}

A total of 206 patients [ $62.1 \%$ males and $37.9 \%$ females] between the ages of 18-67, were included in the study. The effects of demographic data of patients, NSD type, Keros classification, prevalence rates of optic nerve type; as ethmoid air cell variants Agger nasi cell, Onoid cell (Figure 1), Haller cell, supraorbital ethmoid cell (figure 2); as nasal concha variants superior concha bullosa, middle concha bullosa (Figure 3), paradoxal middle concha; as paranasal sinus pneumatization variants frontal and maxillary sinus hypoplasia; and accessory pneumatization variants, uncinate bulla (Figure 4), ACPP (Figure 5), and gender on the distribution of these variants are shown in Table 1.

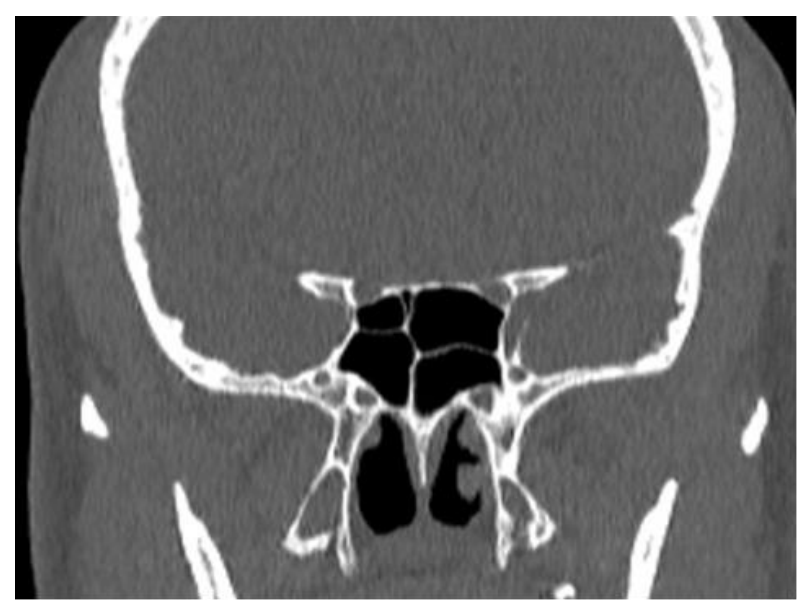

Figure 1. Coronal paranasal CT image of 38-year-old man with bilateral onodi cell (arrows). 


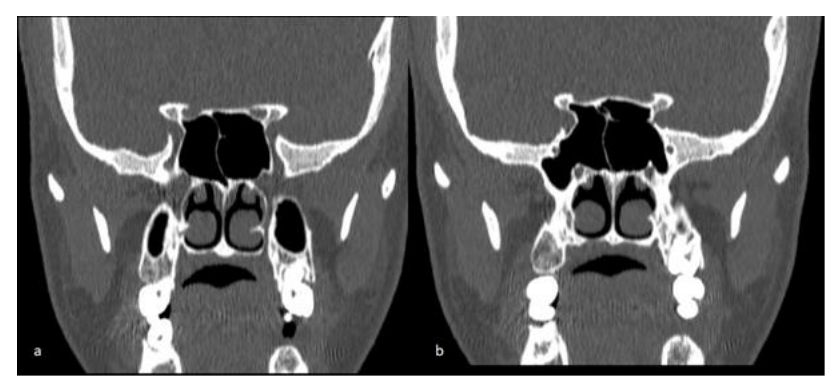

Figure 5. Coronal paranasal CT image of a 30-year- old women with ACPP. a, Coronal CT section showing DELANO TYPE 2 Optic nerve canal (thick arrow). b, Coronal CT section showing ACPP (thin arrow).

Agger nasi was the most frequently paranasal anatomical variation ( $56.8 \%$ ), followed by middle concha bullosa (53.9\%) and ACPP (35.0\%) (Table 1)

The most frequently Keros type was Type 2 (67 $\%)$ and the most frequently seen optic nerve type was Type $1(48.1 \%)$, according to Delano classification. When we look at the effect of gender on Keros and Delano classifications, we saw that there was no significant difference between them $(\mathrm{p}=0.349, \mathrm{p}=$ 0.077) (Table 1). The prevalence of NSD types as per Mladina is significant between males and females ( $\mathrm{p}$ $=0.036$ ). Among males, Type 4 is the most common nasal septum deformity (19.5\%) (Figure 3), whereas Type 7 has the highest prevalence among female patients $(23.1 \%)$. The presence of supraorbital ethmoid is significantly higher in males compared to female patients [21.1\% vs. $6.4 \%](p=0.005)$ (Table $1)$.

There is no significant difference was found between NSD types as per angle classification in terms of the sinonasal variation $(p>0.05)$ (Table 2).

The analysis of correlations between the sinonasal anatomical variations accompanying the septum deviation is shown in Table 3. A significant positive correlation between the presence of Haller cell and onodi cells was observed $(\phi=0.154, \mathrm{p}=$ 0.027). Correlations were also significant between the presence of Frontal sinus hypoplasia and Haller cell $(\phi=-0.142, p=0.042)$, Supraorbital Ethmoid cell $(\phi=-0.173, \mathrm{p}=0.013)$ and Paradoxal middle concha $(\phi=0.152, p=0.029)$. Supraorbital Ethmoid cell was also positively correlated with Uncinate bulla $(\phi=0.152, \mathrm{p}=0.041)$ (Table 3). 
Table 1. Demographic and clinical characteristics of the sample $(n=206)$.

\begin{tabular}{|c|c|c|c|c|}
\hline & Total & $\mathbf{G e}$ & & \\
\hline Patient Characteristics & $\begin{array}{c}\text { median (IQR) or n } \\
(\%)\end{array}$ & Males (n=128) & $\begin{array}{c}\text { Females } \\
(n=78)\end{array}$ & $\mathbf{p}$ \\
\hline \multicolumn{5}{|l|}{ Demographic variables } \\
\hline Age & $27.0(17.8)$ & $29.0(18.0)$ & $27.0(17.8)$ & $0.292^{\mathrm{a}}$ \\
\hline NSD type as per Mladina's classification & & & & $0.036^{b}$ \\
\hline Type 1 & $20(9.7 \%)$ & $12(9.4 \%)$ & $8(10.3 \%)$ & \\
\hline Type 2 & $22(10.7 \%)$ & $14(10.9 \%)$ & $8(10.3 \%)$ & \\
\hline Type 3 & $30(14.6 \%)$ & $13(10.2 \%)$ & $17(21.8 \%)$ & \\
\hline Type 4 & $30(14.6 \%)$ & $25(19.5 \%)$ & $5(6.4 \%)$ & \\
\hline Type 5 & $32(15.5 \%)$ & $22(17.2 \%)$ & $10(12.8 \%)$ & \\
\hline Type 6 & $36(17.5 \%)$ & $24(18.8 \%)$ & $12(15.4 \%)$ & \\
\hline Type 7 & $36(17.5 \%)$ & $18(14.1 \%)$ & $18(23.1 \%)$ & \\
\hline NSD type as per angle classification & & & & $0.117^{b}$ \\
\hline Type 1 & $35(17.0 \%)$ & $18(14.1 \%)$ & $17(21.8 \%)$ & \\
\hline Type 2 & $100(48.5 \%)$ & $69(53.9 \%)$ & $31(39.7 \%)$ & \\
\hline Type 3 & $71(34.5 \%)$ & $41(32.0 \%)$ & $30(38.5 \%)$ & \\
\hline \multicolumn{5}{|l|}{ Variation of ethmoid air cell } \\
\hline Agger nasi cell & $117(56.8 \%)$ & $67(52.3 \%)$ & $50(64.1 \%)$ & $0.098^{\mathrm{b}}$ \\
\hline Onodi cell & $40(19.4 \%)$ & $26(20.3 \%)$ & $14(17.9 \%)$ & $0.677^{b}$ \\
\hline Haller cell & $63(30.6 \%)$ & $44(34.4 \%)$ & $19(24.4 \%)$ & $0.130^{\mathrm{b}}$ \\
\hline Supraorbital Ethmoid cell & $32(15.5 \%)$ & $27(21.1 \%)$ & $5(6.4 \%)$ & $\mathbf{0 . 0 0 5}^{\mathrm{b}}$ \\
\hline \multicolumn{5}{|l|}{ Variation of nasal turbinate } \\
\hline Sup. concha bulloza & $4(1.9 \%)$ & $4(3.1 \%)$ & $0(0.0 \%)$ & $0.300^{\mathrm{c}}$ \\
\hline Middle concha bulloza & $111(53.9 \%)$ & $63(49.2 \%)$ & $48(61.5 \%)$ & $0.085^{\mathrm{b}}$ \\
\hline Paradoxal middle concha & $38(18.4 \%)$ & $26(20.3 \%)$ & $12(15.4 \%)$ & $0.376^{\mathrm{b}}$ \\
\hline \multicolumn{5}{|l|}{ Paranasal sinus pneumatization variations } \\
\hline Frontal sinus hypoplasia & $48(23.3 \%)$ & $30(23.4 \%)$ & $18(23.1 \%)$ & $0.953^{b}$ \\
\hline Maxillary sinus hypoplasia & $7(3.4 \%)$ & $6(4.7 \%)$ & $1(1.3 \%)$ & $0.257^{\mathrm{c}}$ \\
\hline \multicolumn{5}{|l|}{ Accessory pneumatization variations } \\
\hline Uncinate bulla & $18(8.7 \%)$ & $10(7.8 \%)$ & $8(10.3 \%)$ & $0.547^{b}$ \\
\hline Anterior Clinoid Process pneumatization & $72(35.0 \%)$ & $49(38.3 \%)$ & $23(29.5 \%)$ & $0.199^{b}$ \\
\hline Delano Classification & & & & $0.077^{b}$ \\
\hline Type 1 & $99(48.1 \%)$ & $53(41.4 \%)$ & $46(59.0 \%)$ & \\
\hline Type 2 & $73(35.4 \%)$ & $51(39.8 \%)$ & $22(28.2 \%)$ & \\
\hline Type 3 & $16(7.8 \%)$ & $10(7.8 \%)$ & $6(7.7 \%)$ & \\
\hline Type 4 & $18(8.7 \%)$ & $14(10.9 \%)$ & $4(5.1 \%)$ & \\
\hline Keros Classification & & & & $0.349^{\mathrm{b}}$ \\
\hline Type 1 & $39(18.9 \%)$ & $27(21.1 \%)$ & $12(15.4 \%)$ & \\
\hline Type 2 & $138(67.0 \%)$ & $81(63.3 \%)$ & $57(73.1 \%)$ & \\
\hline Type 3 & $29(14.1 \%)$ & $20(15.6 \%)$ & $9(11.5 \%)$ & \\
\hline
\end{tabular}

Data summarized as median (IQR) or frequency (\%). ${ }^{a}$ Mann Whitney U test ${ }^{b}$ Pearson's chi-square test ${ }^{c}$ Fisher's exact test. Bold pvalues indicate statistical significance at $\alpha=0.05$. IQR: Interquartile range. 
Table 2. Comparison of variables between NSD type as per angle classification

\begin{tabular}{|c|c|c|c|c|}
\hline & Tip $1(n=35)$ & Tip $2(n=100)$ & Tip $3(n=71)$ & $\mathbf{p}$ \\
\hline \multicolumn{5}{|l|}{ Demographic variables } \\
\hline Age & $25.5(17.3)$ & $30.5(17.8)$ & $27.0(18.0)$ & $0.575^{\mathrm{a}}$ \\
\hline Gender & & & & $0.117^{\mathrm{b}}$ \\
\hline Male & $18(51.4 \%)$ & $69(69.0 \%)$ & $41(57.7 \%)$ & \\
\hline Female & $17(48.6 \%)$ & $31(31.0 \%)$ & $30(42.3 \%)$ & \\
\hline \multicolumn{5}{|l|}{ Variation of ethmoid air cell } \\
\hline Agger nasi cell & $19(54.3 \%)$ & $56(56.0 \%)$ & $42(59.2 \%)$ & $0.871^{\mathrm{a}}$ \\
\hline Onodi cell & $7(20.0 \%)$ & $23(23.0 \%)$ & $10(14.1 \%)$ & $0.347^{\mathrm{a}}$ \\
\hline Haller cell & $9(25.7 \%)$ & $34(34.0 \%)$ & $20(28.2 \%)$ & $0.567^{\mathrm{a}}$ \\
\hline Supraorbital Ethmoid cell & $8(22.9 \%)$ & $15(15.0 \%)$ & $9(12.7 \%)$ & $0.388^{\mathrm{a}}$ \\
\hline \multicolumn{5}{|l|}{ Variation of nasal turbinate } \\
\hline Sup. concha bulloza & $0(0.0 \%)$ & $4(4.0 \%)$ & $0(0.0 \%)$ & $0.171^{\mathrm{c}}$ \\
\hline Middle concha bulloza & $16(45.7 \%)$ & $55(55.0 \%)$ & $40(56.3 \%)$ & $0.559^{\mathrm{b}}$ \\
\hline Paradoxal middle concha & $5(14.3 \%)$ & $14(14.0 \%)$ & $19(26.8 \%)$ & $0.083^{\mathrm{b}}$ \\
\hline \multicolumn{5}{|l|}{ Paranasal sinus pneumatization variations } \\
\hline Frontal sinus hypoplasia & $8(22.9 \%)$ & $20(20.0 \%)$ & $20(28.2 \%)$ & $0.460^{\mathrm{b}}$ \\
\hline Maxillary sinus hypoplasia & $0(0.0 \%)$ & $4(4.0 \%)$ & $3(4.2 \%)$ & $0.679^{c}$ \\
\hline \multicolumn{5}{|l|}{ Accessory pneumatization variations } \\
\hline Uncinate bulla & $5(14.3 \%)$ & $5(5.0 \%)$ & $8(11.3 \%)$ & $0.159^{\mathrm{b}}$ \\
\hline Anterior Clinoid Process pneumatization & $15(42.9)$ & $30(30.0)$ & $27(38.0)$ & $0.311^{\mathrm{b}}$ \\
\hline Delano Classification & & & & $0.801^{\mathrm{b}}$ \\
\hline Type 1 & $14(40.0 \%)$ & $52(52.0 \%)$ & $33(46.5 \%)$ & \\
\hline Type 2 & $14(40.0 \%)$ & $31(31.0 \%)$ & $28(39.4 \%)$ & \\
\hline Type 3 & $4(11.4 \%)$ & $7(7.0 \%)$ & $5(7.0 \%)$ & \\
\hline Type 4 & $3(8.6 \%)$ & $10(10.0 \%)$ & $5(7.0 \%)$ & \\
\hline Keros Classification & & & & $0.059^{\mathrm{b}}$ \\
\hline Type 1 & $4(11.4 \%)$ & $14(14.0 \%)$ & $21(29.6 \%)$ & \\
\hline Type 2 & $24(68.6 \%)$ & $73(73.0 \%)$ & $41(57.7 \%)$ & \\
\hline Type 3 & $7(20.0 \%)$ & $13(13.0 \%)$ & $9(12.7 \%)$ & \\
\hline
\end{tabular}

Data summarized as median (IQR) or frequency (\%). ${ }^{a}$ Kruskal-Wallis test ${ }^{b}$ Pearson's chi-square test ${ }^{c}$ Fisher's exact test. Bold $p$-values indicate statistical significance at $\alpha=0.05$. IQR: Interquartile range. 


\section{Radiologic Analysis of Sinonasal Anatomical Variations}

Table 3. Correlations between anatomic variants

\begin{tabular}{|c|c|c|c|c|c|c|c|c|c|c|c|c|}
\hline & \multicolumn{3}{|l|}{ Agger nasi } & \multicolumn{3}{|l|}{ Onodi } & \multicolumn{3}{|l|}{ Haller } & \multicolumn{3}{|c|}{ Supraorbital Ethmoid } \\
\hline & Yes & No & $\mathbf{p}$ & Yes & No & $\mathbf{p}$ & Yes & No & $\mathbf{p}$ & Yes & No & $\mathbf{p}$ \\
\hline \multirow[t]{2}{*}{$\begin{array}{l}\text { Agger nasi } \\
\text { cell }\end{array}$} & - & - & - & $\begin{array}{l}21 \\
(17.9 \%)\end{array}$ & $\begin{array}{l}96 \\
(82.1 \%)\end{array}$ & 0.541 & $38(32.5)$ & $79(67.5)$ & $\begin{array}{l}0.49 \\
8\end{array}$ & $\begin{array}{l}18 \\
(15.4 \%)\end{array}$ & $\begin{array}{l}99 \\
(84.6 \%)\end{array}$ & $\begin{array}{l}0.94 \\
6\end{array}$ \\
\hline & & & & $(\phi=-0.043)$ & & & $(\phi=0.047)$ & & & $(\phi=-0.005)$ & & \\
\hline \multirow[t]{2}{*}{ Onodi cell } & $\begin{array}{l}21 \\
(52.5 \%)\end{array}$ & $\begin{array}{l}19 \\
(47.5 \%)\end{array}$ & $\begin{array}{l}0.54 \\
1\end{array}$ & - & - & - & $\begin{array}{l}18 \\
(45.0 \%)\end{array}$ & $\begin{array}{l}22 \\
(55.0 \%)\end{array}$ & $\begin{array}{l}0.02 \\
7\end{array}$ & $5(12.5 \%)$ & $\begin{array}{l}35 \\
(87.5 \%)\end{array}$ & $\begin{array}{l}0.55 \\
5\end{array}$ \\
\hline & $(\phi=0.043)$ & & & & & & $(\phi=0.154)$ & & & $(\phi=-0.041)$ & & \\
\hline \multirow[t]{2}{*}{ Haller cell } & $\begin{array}{l}38 \\
(60.3 \%)\end{array}$ & $\begin{array}{l}25 \\
(39.7 \%)\end{array}$ & $\begin{array}{l}0.49 \\
8\end{array}$ & $\begin{array}{l}18 \\
(28.6 \%)\end{array}$ & $\begin{array}{l}45 \\
(71.4 \%)\end{array}$ & 0.027 & - & - & - & $\begin{array}{l}12 \\
(19.0 \%)\end{array}$ & $\begin{array}{l}51 \\
(81.0 \%)\end{array}$ & $\begin{array}{l}0.35 \\
5\end{array}$ \\
\hline & $(\phi=0.047)$ & & & $(\phi=0.154)$ & & & & & & $(\phi=0.064)$ & & \\
\hline \multirow[t]{2}{*}{$\begin{array}{l}\text { Supraorbital } \\
\text { Ethmoid cell }\end{array}$} & $\begin{array}{l}18 \\
(56.3 \%)\end{array}$ & $\begin{array}{l}14 \\
(43.7 \%)\end{array}$ & $\begin{array}{l}0.94 \\
6\end{array}$ & $5(15.6 \%)$ & $\begin{array}{l}27 \\
(84.4 \%)\end{array}$ & 0.555 & $\begin{array}{l}12 \\
(37.5 \%)\end{array}$ & $\begin{array}{l}20 \\
(62.5 \%)\end{array}$ & $\begin{array}{l}0.35 \\
5\end{array}$ & - & - & - \\
\hline & $(\phi=-0.005)$ & & & $(\phi=-0.041)$ & & & $(\phi=0.064)$ & & & & & \\
\hline \multirow[t]{2}{*}{$\begin{array}{l}\text { Sup. concha } \\
\text { bullosa }\end{array}$} & $3(75.0 \%)$ & $1(25.0 \%)$ & $\begin{array}{l}0.63 \\
5\end{array}$ & $1(25.5 \%)$ & $\begin{array}{l}3 \\
(75.0 \%)\end{array}$ & 1 & $2(50.0 \%)$ & $2(50.0 \%)$ & $\begin{array}{l}0.58 \\
7\end{array}$ & $1(25.0 \%)$ & $3(75.0 \%)$ & 1 \\
\hline & $(\phi=0.052)$ & & & $(\phi=0.020)$ & & & $(\phi=0.059)$ & & & $(\phi=0.037)$ & & \\
\hline \multirow{2}{*}{$\begin{array}{l}\text { Middle } \\
\text { concha } \\
\text { bullosa }\end{array}$} & $\begin{array}{l}63 \\
(56.8 \%)\end{array}$ & $\begin{array}{l}48 \\
(43.2 \%)\end{array}$ & 0.99 & $\begin{array}{l}24 \\
(21.6 \%)\end{array}$ & $\begin{array}{l}87 \\
(78.4 \%)\end{array}$ & 0.387 & $\begin{array}{l}33 \\
(29.7 \%)\end{array}$ & $\begin{array}{l}78 \\
(70.3 \%)\end{array}$ & $\begin{array}{l}0.77 \\
4\end{array}$ & $\begin{array}{l}17 \\
(15.3 \%)\end{array}$ & $\begin{array}{l}94 \\
(84.7 \%)\end{array}$ & $\begin{array}{l}0.92 \\
5\end{array}$ \\
\hline & $(\phi=-0.001)$ & & & $(\phi=0.060)$ & & & $(\phi=-0.020)$ & & & $(\phi=-0.007)$ & & \\
\hline \multirow[t]{2}{*}{$\begin{array}{l}\text { Paradoxal } \\
\text { middle concha }\end{array}$} & $\begin{array}{l}25 \\
(65.8 \%)\end{array}$ & $\begin{array}{l}13 \\
(34.2 \%)\end{array}$ & $\begin{array}{l}0.21 \\
5 \\
\end{array}$ & $9(23.7 \%)$ & $\begin{array}{l}29 \\
(76.3 \%)\end{array}$ & 0.462 & $\begin{array}{l}12 \\
(31.6 \%)\end{array}$ & $\begin{array}{l}26 \\
(68.4 \%)\end{array}$ & $\begin{array}{l}0.88 \\
3 \\
\end{array}$ & $4(10.5 \%)$ & $\begin{array}{l}34 \\
(89.5 \%)\end{array}$ & $\begin{array}{l}0.34 \\
5 \\
\end{array}$ \\
\hline & $(\phi=0.086)$ & & & $(\phi=0.051)$ & & & $(\phi=0.010)$ & & & $(\phi=-0.066)$ & & \\
\hline \multirow[t]{2}{*}{$\begin{array}{l}\text { Frontal sinus } \\
\text { hypoplasia }\end{array}$} & $\begin{array}{l}33 \\
(68.8 \%) \\
\end{array}$ & $\begin{array}{l}15 \\
(31.3 \%) \\
\end{array}$ & $\begin{array}{l}0.05 \\
6 \\
\end{array}$ & $9(18.8 \%)$ & $\begin{array}{l}39 \\
(81.3 \%) \\
\end{array}$ & 0.894 & $9(18.8 \%)$ & $\begin{array}{l}39 \\
(81.3 \%)\end{array}$ & $\begin{array}{l}.04 \\
2 \\
\end{array}$ & $2(4.2 \%)$ & $\begin{array}{l}46 \\
(95.8 \%) \\
\end{array}$ & $\begin{array}{l}0.01 \\
3 \\
\end{array}$ \\
\hline & $(\phi=0.133)$ & & & $(\phi=-0.009)$ & & & $(\phi=-0.142)$ & & & $(\phi=-0.173)$ & & \\
\hline \multirow[t]{2}{*}{$\begin{array}{l}\text { Maxillary } \\
\text { sinus } \\
\text { hypoplasia }\end{array}$} & $3(42.9 \%)$ & $4(57.1 \%)$ & $\begin{array}{l}0.70 \\
1\end{array}$ & $0(0.0 \%)$ & $\begin{array}{l}7 \\
(100.0 \\
\%) \\
\end{array}$ & 0.35 & $0(0.0 \%)$ & $\begin{array}{l}7 \\
(100.0 \%)\end{array}$ & $\begin{array}{l}0.10 \\
3\end{array}$ & $1(14.3 \%)$ & $6(85.7 \%)$ & 1 \\
\hline & $(\phi=-0.053)$ & & & $(\phi=-0.092)$ & & & $(\phi=-0.124)$ & & & $(\phi=-0.006)$ & & \\
\hline \multirow[t]{2}{*}{ Uncinate bulla } & $8(44.4 \%)$ & $\begin{array}{l}10 \\
(55.6 \%)\end{array}$ & $\begin{array}{l}0.26 \\
8\end{array}$ & $3(16.7 \%)$ & $\begin{array}{l}15 \\
(83.3 \%)\end{array}$ & 1 & $6(33.3 \%)$ & $\begin{array}{l}12 \\
(66.7 \%)\end{array}$ & $\begin{array}{l}0.79 \\
1\end{array}$ & $6(33.3 \%)$ & $\begin{array}{l}12 \\
(66.7 \%)\end{array}$ & $\begin{array}{l}0.04 \\
1\end{array}$ \\
\hline & $(\phi=-0.077)$ & & & $(\phi=-0.022)$ & & & $(\phi=0.018)$ & & & $(\phi=0.152)$ & & \\
\hline \multirow{2}{*}{$\begin{array}{l}\text { Anterior } \\
\text { Clinoid } \\
\text { Process } \\
\text { pneumatizatio }\end{array}$} & $\begin{array}{l}41 \\
(56.9 \%)\end{array}$ & $\begin{array}{l}31 \\
(43.1 \%)\end{array}$ & $\begin{array}{l}0.97 \\
5\end{array}$ & $\begin{array}{l}15 \\
(20.8 \%)\end{array}$ & $\begin{array}{l}57 \\
(79.2 \%)\end{array}$ & 0.706 & $\begin{array}{l}24 \\
(33.3 \%)\end{array}$ & $\begin{array}{l}48 \\
(66.7 \%)\end{array}$ & 0.53 & $\begin{array}{l}16 \\
(22.2 \%)\end{array}$ & $\begin{array}{l}57 \\
(77.8 \%)\end{array}$ & $\begin{array}{l}0.05 \\
2\end{array}$ \\
\hline & $(\phi=0.002)$ & & & $(\phi=0.026)$ & & & $(\phi=0.044)$ & & & $(\phi=0.135)$ & & \\
\hline
\end{tabular}

P-values are calculated from Pearson's chi-square or Fisher's exact test. $\phi$ : Phi correlation coefficient. Bold p-values indicate statistically significant correlation at $\alpha=0.05$. 
Table 3. (cont). Correlations between anatomic variants.

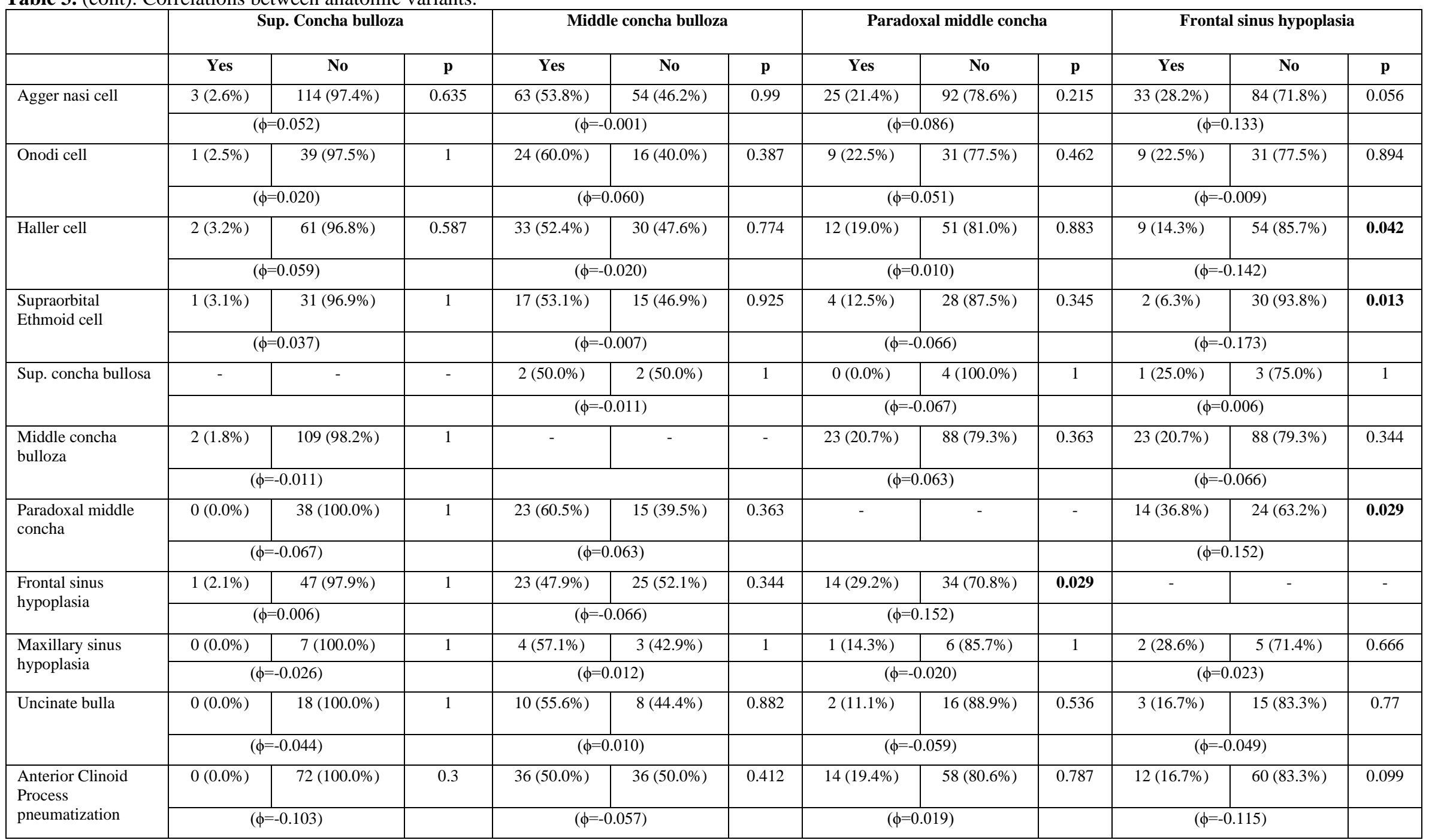

P-values are calculated from Pearson's chi-square or Fisher's exact test. $\phi$ : Phi correlation coefficient. Bold p-values indicate statistically significant correlation at $\alpha=0.05$ 


\section{Radiologic Analysis of Sinonasal Anatomical Variations}

$\underline{\text { Table } 3 \text { (cont). Correlations between anatomic variants. }}$

$\begin{array}{lll}\text { Maxillary sinus hypoplasia } & \text { Uncinate bulla } & \text { Anterior Clinoid Process }\end{array}$

\begin{tabular}{|c|c|c|c|c|c|c|c|c|c|}
\hline & Yes & No & $\mathbf{p}$ & Yes & No & $\mathbf{p}$ & Yes & No & $\mathbf{p}$ \\
\hline \multirow[t]{2}{*}{ Agger nasi cell } & $3(2.6 \%)$ & $114(97.4 \%)$ & 0.701 & $8(6.8 \%)$ & $10(93.2 \%)$ & 0.268 & $41(35.0 \%)$ & $76(65.0 \%)$ & 0.975 \\
\hline & \multicolumn{2}{|c|}{$(\phi=-0.053)$} & & \multicolumn{2}{|c|}{$(\phi=-0.077)$} & \multicolumn{4}{|c|}{$(\phi=0.002)$} \\
\hline \multirow[t]{2}{*}{ Onodi cell } & $0(0.0 \%)$ & $40(100.0 \%)$ & 0.35 & $3(7.5 \%)$ & $37(92.5 \%)$ & 1 & $15(37.5 \%)$ & $25(62.5 \%)$ & 0.706 \\
\hline & \multicolumn{2}{|c|}{$(\phi=-0.092)$} & & \multicolumn{2}{|c|}{$(\phi=-0.022)$} & \multicolumn{4}{|c|}{$(\phi=0.026)$} \\
\hline \multirow[t]{2}{*}{ Haller cell } & $0(0.0 \%)$ & $63(100.0 \%)$ & 0.103 & $6(9.5 \%)$ & $57(90.5 \%)$ & 0.791 & $24(38.1 \%)$ & $39(61.9 \%)$ & 0.53 \\
\hline & \multicolumn{2}{|c|}{$(\phi=-0.124)$} & & \multicolumn{2}{|c|}{$(\phi=0.018)$} & \multicolumn{4}{|c|}{$(\phi=0.044)$} \\
\hline \multirow[t]{2}{*}{$\begin{array}{l}\text { Supraorbital } \\
\text { Ethmoid cell }\end{array}$} & $1(3.1 \%)$ & $31(96.9 \%)$ & 1 & $\begin{array}{c}6 \\
(18.7 \%) \\
\end{array}$ & $26(81.3 \%)$ & 0.041 & $16(50.0 \%)$ & $16(50.0 \%)$ & 0.052 \\
\hline & \multicolumn{2}{|c|}{$(\phi=-0.006)$} & & \multicolumn{2}{|c|}{$(\phi=0.152)$} & \multicolumn{4}{|c|}{$(\phi=0.135)$} \\
\hline \multirow{2}{*}{$\begin{array}{l}\text { Sup. concha bulloza } \\
\text { cell }\end{array}$} & $0(0.0 \%)$ & $4(100.0 \%)$ & 1 & $0(0.0 \%)$ & $4(100.0 \%)$ & 1 & $0(0.0 \%)$ & $4(100.0 \%)$ & 0.3 \\
\hline & \multicolumn{2}{|c|}{$(\phi=-0.026)$} & & \multicolumn{2}{|c|}{$(\phi=-0.044)$} & \multicolumn{4}{|c|}{$(\phi=-0.103)$} \\
\hline \multirow[t]{2}{*}{$\begin{array}{l}\text { Middle concha } \\
\text { bulloza }\end{array}$} & $4(3.6 \%)$ & $107(96.4 \%)$ & 1 & $\begin{array}{c}10 \\
(9.0 \%) \\
\end{array}$ & $\begin{array}{c}101 \\
(91.0 \%)\end{array}$ & 0.882 & $36(32.4 \%)$ & $75(67.6 \%)$ & 0.412 \\
\hline & \multicolumn{2}{|c|}{$(\phi=0.012)$} & & \multicolumn{2}{|c|}{$(\phi=0.010)$} & \multicolumn{4}{|c|}{$(\phi=-0.057)$} \\
\hline \multirow{2}{*}{$\begin{array}{l}\text { Paradoxal middle } \\
\text { concha }\end{array}$} & $1(2.6 \%)$ & $37(97.4 \%)$ & 1 & $2(5.3 \%)$ & $36(94.7 \%)$ & 0.536 & $14(36.8 \%)$ & $24(63.2 \%)$ & 0.787 \\
\hline & \multicolumn{2}{|c|}{$(\phi=-0.020)$} & & \multicolumn{2}{|c|}{$(\phi=-0.059)$} & \multicolumn{4}{|c|}{$(\phi=0.019)$} \\
\hline \multirow{2}{*}{$\begin{array}{l}\text { Frontal sinus } \\
\text { hypoplasia }\end{array}$} & $2(4.2 \%)$ & $46(95.8 \%)$ & 0.666 & $3(6.3 \%)$ & $45(93.8 \%)$ & 0.77 & $12(25.0 \%)$ & $36(75.0 \%)$ & 0.099 \\
\hline & \multicolumn{2}{|c|}{$(\phi=0.023)$} & & \multicolumn{2}{|c|}{$(\phi=-0.049)$} & \multicolumn{4}{|c|}{$(\phi=-0.115)$} \\
\hline \multirow{2}{*}{$\begin{array}{l}\text { Maxillary sinus } \\
\text { hypoplasia }\end{array}$} & - & - & - & $0(0.0 \%)$ & $7(100.0 \%)$ & 1 & $1(14.3 \%)$ & $6(85.7 \%)$ & 0.425 \\
\hline & & & & $(\phi=$ & $0.058)$ & & $(\phi=-($ & .081) & \\
\hline Uncinate bulla & $0(0.0 \%)$ & $40(100.0 \%)$ & 0.35 & $3(7.5 \%)$ & $37(92.5 \%)$ & 1 & $15(37.5 \%)$ & $25(62.5 \%)$ & 0.706 \\
\hline & & $0.092)$ & & $(\phi=$ & $0.022)$ & & $(\phi=0$ & 026) & \\
\hline $\begin{array}{l}\text { Anterior Clinoid } \\
\text { Process }\end{array}$ & $0(0.0 \%)$ & $63(100.0 \%)$ & 0.103 & $6(9.5 \%)$ & $57(90.5 \%)$ & 0.791 & $24(38.1 \%)$ & $39(61.9 \%)$ & 0.53 \\
\hline pneumatization & $(\phi=$ & $.124)$ & & $(\phi$ & 018) & & $(\phi=$ & 44) & \\
\hline
\end{tabular}

P-values are calculated from Pearson's chi-square or Fisher's exact test. $\phi$ : Phi correlation coefficient. Bold p-values indicate statistically significant correlation at $\alpha=0.05$.

There is no significant correlation between the presence of onodi cell and ACPP $(\phi=0.026, \mathrm{p}=$ 0.706) (Figure 6).

A significant association was observed between Delano classification and presence of ACPP ( $p<$ 0.001) (Figure 7). While the majority of the patients with ACPP have Type 2 Delano classification $(n=34$, $47.2 \%$ ) (Figure 5), ACPP was present in all patients in the type 3 Delano group (Figure 7).

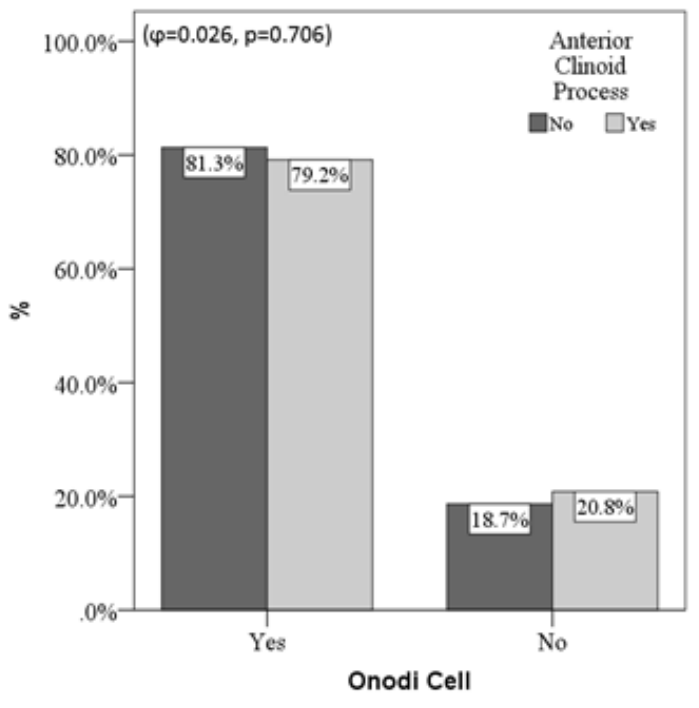

Figure 6. Association between the presence of onodi cell and Anterior Clinoid Process pneumatization 


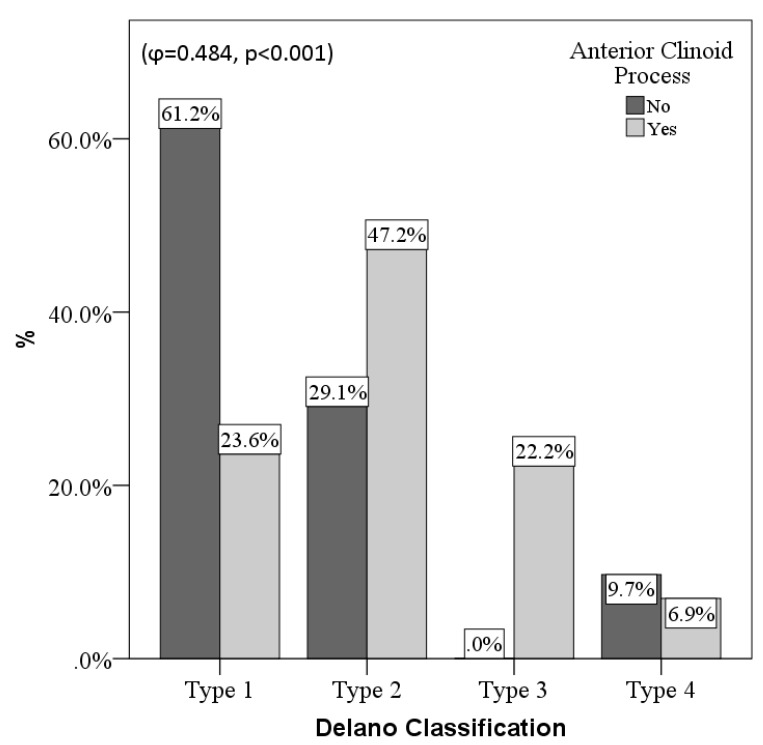

Figure 7. Association between Delano classification and presence of Anterior Clinoid Process Pneumatization.

\section{Discussion}

Various classification systems have been defined for typing NSD in the literature. Mladina et al. (Mladina et al., 2008) defined the Mladina classification for NSD typing and reported that the most frequently type was type 3 . Haytoglu et al. (Haytoglu et al., 2017) reported that type 2 is the most frequently type in patients with septoplasty. In our study, unlike the literature findings, the most frequently NSD type was type 4 in men and type 7 in women. These differences in the literature may result from differences of geographic regions.

Based on the literature, many studies have investigated the frequency of concurrence of concha bullosa with septum deviation, which is one of the sinonasal anatomical variations (Koo et al., 2017; ElTaher et al., 2019). The various incidence rates of concha bullosa with septal deviation have been reported (Yigit et al, 2010) $45.34 \%$, Yazici et al. (Yazici, 2019) $45.3 \%$, Koo et al. (Koo et al., 2017) $52.4 \%$ ). In our study, the incidence of concha bullosa was $53.9 \%$ by these studies previous studies showing that the NSD angle is related to the concha bullosa (Uygur et al., 2003; El-Taher et al., 2019). Contrary to these findings, any relationship between the NSD angle, and concha bullosa was not found in our study.

The incidence of paradoxal middle turbinates, which is one of the anatomical variations of turbinates, ranges from 3 to $40 \%$ in the literature (Bolger et al., 1991; Ozcan et al., 2008). In our study, paradoxal middle turbinate incidence was $18.4 \%$ and this rate was compatible with the literature.

The prevalence of the Haller cell, which is one of the ethmoid air cell variants, varies between $2.7-$
$45.1 \%$ in the literature (Bolger et al., 1991; Yazici, 2019). Shokri et al. (Shokri et al., 2019) found a correlation between Haller cell and NSD. In our study, the incidence of the Haller cell was $30.6 \%$. We found a significant correlation between Haller cell and Onodi cell and frontal sinus hypoplasia. The presence of sinonasal anatomical variants and correlations between them are important in order to establish a diagnosis and treatment plan and to prevent possible surgical complications.

The prevalence of supraorbital ethmoid cell, which is important for safe dissection of the frontal ostium, varies in the literature by ethnic origin (Zhang et al., 2007). The incidence of supraorbital ethmoid cell has been reported as $5.3 \%$ and its association with the anterior ethmoid artery has been shown (Zhang et al., 2007). In our study, the incidence of supraorbital ethmoid cells was $15.5 \%$ and it was significantly higher in men compared to women [27 $(21.1 \%)$ vs. $5(6.4 \%)]$. We also found a significant correlation between supraorbital cell, paradoxal middle turbinate and uncinate bulla. The prevalence of uncinate bulla, which is a rare anatomic variant that can lead to narrowing of the infundibulum, varies between 0.4-13\% in the literature (Fadda et al., 2012; Yazici, 2019). In our study, the incidence of uncinate bulla was $8.7 \%$ and it were in concordance with the literature.

In order to prevent vital complications that may occur during transsphenoidal and skull base surgery, it is essential to identify anatomic variations associated with the SS preoperatively. Removal of the ACPP during skull base surgery carries the risk of cerebrospinal fluid rhinorrhea and associated sepsis (Nandapalan et al., 1996; Sirikci et al., 2000). Prior to skull base surgery, the risk of this complication is reduced by identifying ACPP in paranasal CT and preferring extradural approach (Noguchi et al., 2005). In the literature, the prevalence rates of ACPP, and Onodi cell were reported to range between $13.3-16$ $\%$, and $0-18 \%$, respectively $(17,23,25)$ (Bolger et al., 1991; Sirikci et al., 2000; Unal et al., 2006). In a study of patients with NSD, Onodi cells, and ACPP were seen in $2 \%$, and $29.3 \%$ of the patients respectively. In our study, Onodi cells and ACPP were seen in $19.4 \%$, and $35 \%$ of the patients, respectively which were higher when compared with the literature findings. In NSD patients, changes in the turbulence of airflow through the nasal cavity or genetic mechanisms may be associated with pneumatization anomalies. Pneumatization of the anterior clinoid process may cause the optic nerve to medialize into the SS during the development process. Studies have reported that ACPP is 
associated with optic nerve protrusion (Sapci et al., 2004; Itagi et al., 2017). In our study, we identified a positive correlation between ACPP and Delano type 2 and 3, where the optic nerve protrudes into the SS. Especially in patients with NSD, the identification of ACPP by examining paranasal CTs in detail before transsphenoidal and skull base surgery reduces the risks of complications that may occur.

A limitation of the present study is that it was retrospective and not having a control group of patients without septum deviation. Further studies including large numbers of case-control studies are necessary to evaluate the relationship of septal deviation with sinonasal anatomic variants.

\section{Conclusion}

In our study, the incidence of onodi cell, supraorbital ethmoid cell, anterior clinoid process pneumatization were more frequent in patients with nasal septal deviation compared to the literature. However, there is no significant difference was found between NSD types as per angle classification in terms of the presence of sinonasal variation. Careful examination of paranasal CTs before craniomaxillofacial surgeries is important to determine sinonasal anatomic variants, to determine the appropriate treatment plan and to prevent possible complications preoperatively.

Ethics Committee Approval: This retrospective study was performed after approval of the Abant Izzet Baysal University ethics committee in accordance with the Helsinki declaration (07.03. 2019 2019/63).

Peer-review: Externally peer-reviewed.

Author Contributions: Concept -E.K A.G E.D Design E.K E.D Z.C; Audit E.K E.D A.G AU.; Materials - E.K E.D Z.C Data Collection and / or Processing - E.K O.K A.U Analysis and / or Interpretation - E.K O.K A.G Z.C ; Literature review - E.K A.G E.D Z.C.; Text - E.K E.D O.K; Critical Review - E.K A.U O.K

Conflict of Interest: No conflict of interest was declared by the authors.

Financial Disclosure: The authors declared that this study hasn't received no financial support.

\section{References}

Akoglu E, Karazincir S, Balci A, Okuyucu SS, Sumbas H, Dagli AS. Evaluation of the turbinate hypertrophy by computed tomography in patients with deviated nasal septum. Otolaryngol - Head Neck Surg 2007;136:380-4. https://doi.org/10.1016/j.otohns.2006.09.006.

Alkire BC, Bhattacharyya N. An assessment of sinonasal anatomic variants potentially associated with recurrent acute rhinosinusitis. Laryngoscope 2010;120:631-4. https://doi.org/10.1002/lary.20804.

Bolger WE, Parsons DS, Butzin CA. Paranasal sinus bony anatomic variations and mucosal abnormalities: CT analysis for endoscopic sinus surgery. $\quad$ Laryngoscope 1991. https://doi.org/10.1288/00005537-19910100000010.

Delano MC, Fun FY, Zinreich SJ. Relationship of the optic nerve to the posterior paranasal sinuses: A CT anatomic study. Am J Neuroradiol 1996. https://doi.org/10.1016/s0196-0709(97)90018-1.

El-Taher M, AbdelHameed WA, Alam-Eldeen MH, Haridy A. Coincidence of Concha Bullosa with Nasal Septal Deviation; Radiological Study. Indian J Otolaryngol Head Neck Surg 2019. https://doi.org/10.1007/s12070-018-1311-x.

Elahi MM, Frenkiel S, Fageeh N. Paraseptal structural changes and chronic sinus disease in relation to the deviated septum. J Otolaryngol 1997.

Fadda GL, Rosso S, Aversa S, Petrelli A, Ondolo C, Succo G. Multiparametric statistical correlations between paranasal sinus anatomic variations and chronic rhinosinusitis. Acta Otorhinolaryngol Ital 2012;32:244-51.

Haytoglu S, Dengiz R, Muluk NB, Kuran G, Arikan OK. Effects of septoplasty on olfactory function evaluated by the Brief Smell Identification Test: A study of 116 patients. Ear, Nose Throat J 2017. https://doi.org/10.1177/01455613170960101123.

Itagi RM, Adiga CP, Kalenahalli K, Goolahally L, Gyanchandani $M$. Optic nerve canal relation to posterior paranasal sinuses in indian ethnics: Review and objective classification. J Clin Diagnostic Res 2017. https://doi.org/10.7860/JCDR/2017/23447.9510.

Kantarci M, Karasen RM, Alper F, Onbas O, Okur A, Karaman A. Remarkable anatomic variations in paranasal sinus region and their clinical importance. Eur J Radiol 2004;50:296-302. https://doi.org/10.1016/j.ejrad.2003.08.012. 
Koo SK, Kim JD, Moon JS, Jung SH, Lee SH. The incidence of concha bullosa, unusual anatomic variation and its relationship to nasal septal deviation: A retrospective radiologic study. Auris Nasus Larynx 2017;44:561-70. https://doi.org/10.1016/j.anl.2017.01.003.

Mladina R, Čujić E, Šubarić M, Vuković K. Nasal septal deformities in ear, nose, and throat patients. An international study. Am J Otolaryngol - Head Neck Med Surg 2008. https://doi.org/10.1016/j.amjoto.2007.02.002.

Nandapalan V, Watson ID, Swift AC. Beta-2transferrin and cerebrospinal fluid rhinorrhoea. Clin Otolaryngol Allied Sci 1996. https://doi.org/10.1111/j.13652273.1996.tb01737.x.

Noguchi A, Balasingam V, Shiokawa Y, McMenomey SO, Delashaw JB. Extradural anterior clinoidectomy. Technical note. J Neurosurg 2005. https://doi.org/10.3171/jns.2005.102.5.0945.

Ozcan KM, Selcuk A, Ozcan I, Akdogan O, Dere H. Anatomical Variations of Nasal Turbinates. J Craniofac Surg 2008. https://doi.org/10.1097/SCS.0b013e318188a29d.

Sapci T, Derin E, Almaç S, Cumali R, Saydam B, Karavus M. The relationship between the sphenoid and the posterior ethmoid sinuses and the optic nerves in Turkish patients. Rhinology 2004.

Shokri A, Faradmal MJ, Hekmat B. Correlations between anatomical variations of the nasal cavity and ethmoidal sinuses on cone-beam computed tomography scans. Imaging Sci Dent 2019;49:103-13. https://doi.org/10.5624/isd.2019.49.2.103.

Sirikci A, Bayazit YA, Bayram M, Mumbuc S, Gungor K, Kanlikama M. Variations of sphenoid and related structures. Eur Radiol 2000. https://doi.org/10.1007/s003300051016.

Unal B, Bademci G, Bilgili YK, Batay F, Avci E. Risky anatomic variations of sphenoid sinus for surgery. Surg Radiol Anat 2006. https://doi.org/10.1007/s00276-005-0073-9.

Uygur K, Tuz M, Dogru H. The correlation between septal deviation and concha bullosa. Otolaryngol Head Neck Surg 2003. https://doi.org/10.1016/S0194-5998(03)00479-0.

Wuister AMH, Goto NA, Oostveen EJ, De Jong WU, Van Der Valk ES, Kaper NM, et al. Nasal endoscopy is recommended for diagnosing adults with chronic rhinosinusitis. Otolaryngol - Head Neck Surg (United States) 2014;150:359-64. https://doi.org/10.1177/0194599813514510.
Yazici D. The analysis of computed tomography of paranasal sinuses in nasal septal deviation. $\mathbf{J}$ Craniofac Surg 2019;30:E143-7. https://doi.org/10.1097/SCS.0000000000005077.

Yigit O, Acioglu E, Cakır ZA, Sisman AS, Barut AY. Concha bullosa and septal deviation. Eur Arch Oto-Rhino-Laryngology $\quad$ 2010;267:1397-401. https://doi.org/10.1007/s00405-010-1228-9.

Zhang L, Han D, Ge W, Tao J, Wang X, Li Y, et al. Computed tomographic and endoscopic analysis of supraorbital ethmoid cells. Otolaryngol - Head Neck Surg 2007. https://doi.org/10.1016/j.otohns.2007.06.737. 